\title{
Relationships among social networking behaviors, depressive symptom and sedentary behaviors in older adults
}

\author{
Surinrat Baurangtheinthong ${ }^{1}$, Suparb Aree-Ue ${ }^{2 \star}$ and Sirirat Leelacharas ${ }^{2}$ \\ ${ }^{1}$ Master of Nursing Science Program (Gerontological Nursing), Ramathibodi School of Nursing, Faculty of Medicine \\ Ramathibodi Hospital, Mahidol University, Bangkok, 10400, Thailand. \\ ${ }^{2}$ Division of Adult and Gerontological Nursing, Ramathibodi School of Nursing, Faculty of Medicine Ramathibodi \\ Hospital, Mahidol University, Bangkok, 10400, Thailand.
}

Accepted 26 December, 2019

\begin{abstract}
Social networking may help older adults to decrease emotional distress and depression. However, over using or addiction to social networking may lead to sedentary behavior. This descriptive research aimed to examine the relationship between social networking behavior, depressive symptom, and sedentary behavior in older adults. A total of 152 community-dwelling older adults who were living in Bang Bua Thong District, Nonthaburi Province, Thailand, and met inclusion criteria were randomly selected with multi-stage sampling to participate in this study. The data were collected by using The Demographic Questionnaire, Social Network Behavioral Questionnaire, Thai Geriatric Depression Scale-15, and Physical Activity Record Form. Data were analyzed by using descriptive statistics and Spearman's Rank Correlation Coefficient. A significant level was set at .05. Results revealed that the majority of participants had a high level of social networking behavior (48.0\%), but mostly had no depression (92.8\%). There was only $3.3 \%$ of them had sedentary behavior. Regarding the correlation among study variables, social networking behavior had a mild negatively significant relationship with depressive symptoms, but it was not significant relationship to sedentary behavior. Depressive symptoms had a mild negatively significant relationship with sedentary behavior. The result of this study provides healthcare providers to understand social networking health behavior, depressive symptom, and sedentary behavior, and to help the healthcare providers to plan any intervention to promote healthy behavior in older adults.
\end{abstract}

Keywords: Social networking behaviors, depressive symptom, sedentary behavior, older adults.

${ }^{*}$ Corresponding author. E-mail: suparb.are@mahidol.ac.th.

\section{INTRODUCTION}

Currently, in the era of information technology, communication shared via networks of internet systems (social networks) is essential. These networks build interaction among groups of people and are a part of daily life in every age group including older adults. According to using social networks in older adults aged over 65 years and above in the US increased from only 2\% in 2005 up to 35\% in 2015 (Perrin, 2015). In Thailand, older adults aged 60-64 years old were the group of older people who used social networks at most; and men used social networks 3 times higher than women. Moreover, not only older adults living in Bangkok, but also those living in the vicinity areas to Bangkok have used social networks. For example, older adults residing in Nonthaburi were accounted for the $13^{\text {th }}$ range of Thailand; the prevalence rate of using social networks was $8.6 \%$, which was considered for the $4^{\text {th }}$ range of the country (National Statistical Office Ministry of Information and Communication Technology, 2015).

There was a study reporting that $3.3 \%$ of Thai older adults used the internet and the average use of the social networks was from 1 to 3 hours a day; most used devices, like smartphones and tablets in the forms of email, e-learning, or chatting through the program and 
application. Furthermore, the duration in social networks helped older adults to understand themselves and society and helped loneliness decreased, which might decrease depressive symptoms (Loipah, 2011). However, the use of long-lasting social networks could affect older adults' health. A study revealed that social network was related to a decrease of having physical activities (M'hiri et al., 2015). Consequently, they would be sick and had signs and symptoms of metabolic syndromes (De Rezende et al., 2014). Furthermore, they tended to less go out with friends leading them to have depressive symptoms, especially the older adults having mental problems. The depressive symptoms considered a mental health problem, which leads to a reduction in quality of life, and it is the significant cause of depression and the older adults' suicide. According to a survey, the depressive symptom was the mental health problem in older adults aged 55-74 years (WHO, 2017). Moreover, the depressive symptom found in the older adults was different from in other ages as they had physical symptoms before they had mental symptoms (Rodda et al., 2011). The early signs of depressive symptoms may include not having an appetite, weight loss, and change in sleeping behavior and would have other illnesses such as fatigue, tiredness, dysphagia, and palpitation. The diagnosis of depressive symptoms might be underestimated or neglected because the symptoms in older adults were not specific. Once the depressive symptoms were more severe, patients might show obvious signs together with psychological problems including paranoid, hallucination, refusing to attend any activity, awareness deficit, and memory loss. Those symptoms led to secluding, being introvert, and being unable to perform their daily routines leading to the decline in the physical activities and the increase in the risk of death (Bishwajit et al., 2017).

Having a low physical activity or low mobility is the use of the energy in doing the activities less than 500 kilocalories a week, which is considered sedentary behaviors (Carmpbell, 2012). These are related to people having behaviors such as sitting, playing the computer, and watching television. When individuals have sedentary behaviors for a long time, it affects metabolism to work slowly, which causes high blood sugar, blood pressure, and lipid metabolism depleted from the normal stage of bodily systems. Consequently, it could cause diabetes, cardiovascular diseases, and some types of cancers. Furthermore, sedentary behavior is related to emotion and mind, loneliness, and depressive symptom (Rosenberg et al., 2015). These were considered as the health risk in older adults.

At present, technology becomes a part of the older adults' lifestyle the same as other ages. If the older adults spend a long time or addict to social network media, it may increase more sitting behavior as well as depressive symptoms. From evidence as mentioned above, the behaviors of using social networks, depressive symptoms, and sedentary behaviors are related; there was no published study on this matter in Thai older adults' context. Therefore, results from this study are expected to have fundamental knowledge that would help develop any nursing interventions to promote health in older adults' especially suitable physical activities for the era of digital change. The study aim was to explore the relationships among social network behaviors, depressive symptom, and sedentary behaviors in community-dwelling older adults.

\section{MATERIALS AND METHODS}

The descriptive study was employed. The population was older adults aged 60 years and above living at Bang Bua Thong District, Nonthaburi Province who met the following criteria: using online social network at least 1 application, having their own communication equipment, having experience in social networking on communication equipment at least 20 hours since they started using it, having cognitive intact, and being able to communicate, speak, read, and write in Thai. The prospective participants who were being bedridden and having a medical record in psychopaths or schizophrenia were excluded. In determining sample size in this study, the researcher calculated by using $G^{*}$ Power 3.0.10 program (Faul et al., 2009). The researcher configured power as .80, statistical significance value $(\alpha)$ at level .05 , and effect size value as .30 , which was calculated from the previous study findings (Elliot et al., 2014; Oner and Arslantas, 2016). Based on the program calculation, the study samples were 138 participants. However, incompletion data due to incomplete information or withdrawal during data collection, a threat to validity should be avoided by adding the sample more at $10 \%$. Finally, a total of 152 participants was recruited in this study.

\section{Instrument}

The research instrument consisted of two parts including: screening instruments and the instrument for collecting data.

\section{Screening instruments}

Mini-Cog test: This was used for screening older adults' cognitive. The Mini-Cog test was developed by Borson et al. (2003) and translated into Thai and modified by Trongsakul (2013). It consists of 2 parts: repeating 3 words and drawing a clock time at 11.10 a.m. The total score obtained from these two parts is 5 full points; the score obtained less than 3 means that the participants may be cognitive impairment. The validity and reliability were reported as an acceptable value (Trongsakul, 2013).

Questionnaire about social networking: This was developed by the researcher with a 7-item checklist. The questions asked about the experiences in social networks, the frequency or time spent in each day, device, website service, knowing social network website, and quantity of social network friends on the website used. The content validity was confirmed by 3 experts, and content validity index was .96.

\section{Instruments for collecting data}

Demographic data questionnaire: This consisted of 10 items including sex, age, weight, body mass index (BMI), marital status, educational level, average income, underlying disease, and family history of depression. There were 6 closed-ended questions and 4 
open-ended questions.

Social networking behavioral questionnaire (SNBQ): Developed by Senkaew (2015) was a 5-point Likert scale with a total of 10 items. The scoring criteria are ranged from 1 (Lowest) to 5 (Highest) points; a total score is between 5 and 50 The SNBQ was tested for its reliability in 20 older adults (a pilot study) whose characteristics were similar to the main study (152 older participants). The Cronbach's alpha was .70 and .80 , respectively.

Thai geriatric depression Scale-15 (TGDS-15): was translated into Thai by Wongpakaran et al. (2013). There were 15 questions divided into 5 positive questions and 10 negative questions. The total scores would be in the range from 0 to 15 . The total scores $\geq 5$ indicated depressive symptom, and high scores indicated older adults had a high tendency to have depressive symptoms. After translation, it was used to test for reliability in 30 older adults; the internal consistency reliability was .75. In this current study, the Kuder-Richardson (KR-20) of 152 participants was .73.

Physical activity record form: This was used to measure physical activity of the older adults in the past 7 days (Poolsawat, 2007). It was modified from the Physical Activity Scale for the Elderly (PASE) of New England Research Institutes (NERS) (Thiengtham, 2015). This questionnaire was a 28-item composed of 3 parts including activity of daily living, leisure activity, and exercise. The result values were calculated from older adults' energy spent within 7 days. Those who used the low energy of doing activities were considered as having sedentary behaviors. The content validity of this form was confirmed by 4 experts, and the reliability was tested in 25 older adults living in the city; the Cronbach's alpha was .82 (Poolsawat, 2007). In this study, the reliability of Cronbach's alpha was .79.

\section{Ethical consideration}

This study was approved by the Human Research Ethics Committee of Faculty of Medicine Ramathibodi Hospital, Mahidol University (Protocol no. 12-61-74), and requested permission in collecting data in Nonthaburi Provincial Public Health. All participants were provided information including study purposes, confidentiality, and benefits associated with the study before signing the inform consent. The participants were informed of their rights that they could withdraw throughout the study without penalty.

\section{Data collection and analysis}

Data collection was carried out after the study was approvedby the Institutional Review Broad and Graduate Studies, Mahidol University. The prospective older participants were recruited by randomly selected with multi-stage sampling; older participants living in four villages from 3 sub-districts who met inclusion criteria were approached. The eyesight problems may impact the readability of the questionnaire; the interview was used for data collection. The completed data were analyzed by using descriptive statistics for all study variables, and Spearman's rank correlation coefficient was employed for detecting the correlation among study variables since the study variables were not a normal distribution.

\section{RESULTS}

\section{Demographics}

A total of 152 participants were aged between 60 and 92 years, and the average age was $67 \pm 6.11$ years. The majority were in $60-70$ years. Nearly half of them $(49.3 \%)$ were married. More than one-third (36.2\%) had an elementary school, and more than half of them $(53.3 \%)$ had an average income of 500-1,000 baht/month. Moreover, more than half of the participants $(73.3 \%)$ had the underlying disease (s). The first three common underlying diseases were hypertension (46.1\%), dyslipidemia (38.2\%), and diabetes (21.1\%). The participants with 1 to 2 underlying disease (s) were $60.6 \%$. Besides, most participants (45.4\%) had BMI at the obese level. Most of them $(82.9 \%)$ had frequency in social networks 2 to 3 times per day, and the majority $(79.6 \%)$ spent less than 1 hour per time. Moreover, most use was smartphone (98.0\%). The most popular applications were LINE (98.7\%) following Facebook $(54.6 \%)$. The participants learned how to access applications by their friends and their children (80.9\%), and the most average quantity of friends on the website was 11 to 50 people $(35.5 \%)$.

\section{Social networking behaviors, depressive symptom, and sedentary behaviors}

Table 1 illustrates the study variables. The overall social network behaviors of older adults had the average scores at $3.8 \pm 0.2$ scores considered high social network behaviors $(48.0 \%)$. For depressive symptoms, the overall depressive symptom of older adults had the average scores of $1.3 \pm 1.2$ scores considered as no depressive symptom $(92.8 \%)$.

Overall, $88.1 \%$ of participants had enough physical activities with the average energy used of 2,296.3 \pm 934.1 kilocalories a week. However, of all participants, $11.9 \%$ of them did not have enough physical activities.

\section{Relationships among social networking behaviors, depressive symptom, and sedentary behaviors}

The overall social networking behaviors had mild negative significant relationship to depressive symptom $\left(r_{s}=-.170, p<.05\right)$, but there was not significant relationship to sedentary behaviors $\left(r_{s}=.075, p>.05\right)$. In addition, depressive symptom had mild negative significant relationship with sedentary behaviors $\left(r_{s}=-\right.$ $.263, \mathrm{p}<.001)$ (Table 2).

\section{DISCUSSION}

Social networking behaviors, depressive symptom, and sedentary behaviors

Most participants had social network scores at a high level, while about one-third of the participants had social network scores at a moderate level. The current findings 
Table 1. Illustrates study variables $(n=152)$.

\begin{tabular}{lcccc}
\hline Variables & Possible score & Actual score & Mean + SD & $\mathbf{n}(\%)$ \\
\hline Social network behaviors & $1.00-5.00$ & $1.40-4.90$ & $3.6 \pm 0.6$ & $152(100.0)$ \\
Lowest & $1.00-1.80$ & $1.40-1.80$ & $1.6 \pm 0.2$ & $3(2.0)$ \\
Low & $1.81-2.60$ & $2.40-2.60$ & $2.5 \pm 0.1$ & $6(3.9)$ \\
moderate & $2.61-3.40$ & $2.70-3.40$ & $3.1 \pm 0.1$ & $41(27.0)$ \\
High & $3.41-4.20$ & $3.50-4.20$ & $3.8 \pm 0.2$ & $73(48.0)$ \\
Highest & $4.21-5.00$ & $4.30-4.90$ & $4.4 \pm 0.1$ & $29(19.1)$ \\
Depressive symptom & $0-15$ & $0-11$ & & $1.8 \pm 2.0$ \\
No & $0-4$ & $0-4$ & $1.3 \pm 1.2$ & $152(100.0)$ \\
Having the sign & $5-10$ & $5-10$ & $7.3 \pm 2.0$ & $141(92.8)$ \\
Yes & $11-15$ & 11 & $11 \pm 0.0$ & $10(6.6)$ \\
& & & & $1(0.7)$ \\
Physical activity & $<500-\geq 1000$ & $396.5-6,219.9$ & $2,103.4 \pm 1,025.7$ & $152(100.0)$ \\
Enough & $\geq 1000$ & $1,025.9-6,219.9$ & $2,296.3 \pm 934.1$ & $134(88.1)$ \\
Low & $<1000$ & $509.9-993.6$ & $754.3 \pm 172.0$ & $13(8.6)$ \\
Sedentary behaviors & $<500$ & $396.5-487.5$ & $440.9 \pm 41.9$ & $5(3.3)$ \\
\hline
\end{tabular}

Table 2. The Spearman rank correlation coefficient among social networking behaviors, depressive symptom, and sedentary behaviors $(\mathrm{N}=152)$.

\begin{tabular}{lccc}
\hline Variables & $\mathbf{1}$ & $\mathbf{2}$ & $\mathbf{3}$ \\
\hline 1. Social networking behaviors & 1.000 & & \\
2. Depressive symptom & $-.170^{\star}$ & 1.000 & \\
3. Sedentary behaviors & .075 & $-.263^{* *}$ & 1.000 \\
\hline
\end{tabular}

${ }^{*} p<.05,{ }^{* *} p<.001$.

were partly similar to the previous study (Koocharoensin, 2016) investigating social media behaviors in Thai older adults in Chonburi, which revealed that the overall scores of social media behaviors were a moderate level, and the scores of the benefit of social media aspect were high. It can be said that social network behaviors in older adults had varieties and had an impact on their daily life. The reason may be because older adults living in Bangkok and its vicinity including Nonthaburi may be easy to access social networks led them to satisfaction in the social network uses. Besides, using social networks lead older adults to communicate with each other freely, have benefits from the services available via the networks, exchange their opinions, be accepted and respected by friends in networks. As a result, satisfaction in using social networks affects social network behaviors in older adults (Buntadthong, 2014). Moreover, most of the older participants in this study were young-old and were married. These findings are relevant to Gell et al. (2015), who found that these older adult groups used communication networking skills at a high level. However, the use of communication networking skills decreased in older adults who were disabled and visual and cognitive impaired. In this study, there were no older adults having difficulty in using the social network, as a result, they had a high level of social network behaviors.

Depressive symptoms in the participants were found that most of them were considered as no depressive symptoms; however, only $6.6 \%$ indicated the sign of the depressive symptom. It could be implied that the older participants had good mental health. This finding might be because most participants were young to old older adults (the average ages were $67 \pm 6.1$ years old). Similarly to a previous study reported that the occurrence of the mental health problems in older adults in the US was mostly found in the late older adults (Reynolds et al., 2015). Another reason may be that most of them were married. This could be interpreted that living with their spouse or child had more chance to relax as they had social support.

The older adults used the average energy in doing activities in a week at 2,103.4 kilocalories per week; among these participants, $88.1 \%$ of them had enough physical activity, while $8.6 \%$ had low physical activities and $3.3 \%$ had sedentary behaviors. The participants used their highest average energy in leisure activity (839.4 kilocalories per week) following by activity of daily living (794.9 kilocalories per week), and the least energy 
used activity was the exercise (469.0 kilocalories per week). This finding is relevant to the findings of the Institute for Population and Social Research, Mahidol University (2016). In the survey regards physical activities and sedentary behaviors in Thai people in 2012-2016, more than half of the older adults (70\%) had enough physical activities, and most of them were young-older adults (60 to 69 years old). They were able to perform self-care ability and household activities such as cleaning a house and working. Consequently, they still had enough physical activities. However, the least energy used in exercising may decrease due to their age-related decline. When getting old, their physical abilities tended to decline or develop co-morbidities leading older adults to face with a barrier when exercising; they might less perform exercising. According to Akeplakorn (2015), the old-older adults (70 to 79 years old) and the oldest-older adults ( 80 years old and above) were the groups tended to have inadequately physical activities. Nonetheless, the study participants were the older adults living in the big city and its vicinity; most activities they spent were a leisure activity. They spent time watching television and listening to music at $89.5 \%$ as well as sleeping or having daytime rest at $68.4 \%$. These led sedentary behaviors were found in this study although only $3.3 \%$ was observed.

\section{Relationships among social networking behaviors, depressive symptom, and sedentary behaviors}

Overall, social network behaviors had mild negatively significant relationships to depressive symptoms $\left(r_{\mathrm{s}}=-\right.$ $.170, p<.05$ ). It could be concluded that older adults who had social network behaviors tended to have a less depressive symptom. This finding is relevant to the study of Cotton et al. (2014) reporting in 8,000 older adults. Most of those older adults used the internet or social media; consequently, one-third of them had a less depressive symptom when compared with those who did not use it. Moreover, a study of Chopik (2016) about the benefits of using technologies in 591 older adults whose average age was 68 years revealed that older adults who had a positive attitude with social networks had a negatively significant relationship with depressive symptoms $(r=-.09, p<.05)$. The explanation may be that social network could reduce depressive symptom because of social encouragement as findings from previous studies supported that social networks could decrease loneliness, increase satisfaction in life, and bring mental bliss (Chopik, 2016; Heo et al., 2015). Similar to the study of Pomnu and Roonpho (2017), which found that older adults who had more social network behaviors increased their roles and the relationships in the family, leading them to have better mental health. Additionally, using social networks were not only for seeking information, but also for maintaining personal relationships and being a part of the group of friends and the older adults' society (Loipah, 2011; Chartprasert, 2013). These might make the older adults feel satisfied and decreased depressive symptom, congruence with the idea of social integration to health, which indicated that the social network affected the mind, made people to realize in and respected themselves, could face with the problems, had the good quality of life, and reduced the depressive symptom (Berkman et al., 2000).

Depressive symptoms had mild negatively significant relationship with sedentary behaviors $\left(r_{s}=-.263\right.$; $p<$ .001). It means that the older adults who had serious depressive symptoms tended to have sedentary behaviors. Likewise, the study of Schuch et al. (2017) was found that patients who had depressive symptoms had a reduction in physical activities. Similar to another study, which indicated that feeling dismal, depressed, and isolated from the society in the older adults were the predicting factors of gait speed (Shankar et al., 2017). Moreover, Thai older adults with depressive symptoms spent more time walking speed 6 meters than those without depressive symptoms. These findings could be explained that the depressive symptom causes the change of the hypothalamic-pituitary-adrenal axis. As a result, there is an inflammation that produces high IL-6 TNI-2 and C-reactive protein (CRP). Consequently, it affects the mobility and causes the sedentary condition and the reduction in physical activities (Penninx et al., 2003).

However, social network behavior was not significantly related to sedentary behaviors. It was different from the study in other countries as it was found that social networks had a negatively significant relationship with the physical activities (Pearson et al., 2014; Vallance et al., 2016). This might be because, in this study, almost half of the older adults (48\%) had social networking behaviors at a high level. That nearly the same percentage of participants $(45.4 \%)$ who had body mass index (BMI) was the obese level, while there were only $3.3 \%$ had sedentary behaviors. The sedentary conditions might be caused by obesity that made the older adults difficulty to perform physical activities, namely standing or walking that they might not have the problem from their social network behaviors. Related to the previous study (Gell et al., 2015), the sedentary behaviors were caused by physical inabilities related to the increase of age and disability. When considering social network behaviors, most participants $(82.9 \%)$ had the frequency in social network 2-3 times a day, while $79.6 \%$ spent an average time less than 1 hour per time. As a result, it might say that they were not addicted to online media resulting in that no relationship with sedentary behaviors was found in this study. Moreover, when considering activities classified as sedentary behaviors, it was found that watching television and listening to the music used the average energy at $216.1 \pm 205.6$ kilocalories a week, and 
most of them spent the average time more than 1 hour a day. This is relevant to the systematic study about the sedentary behaviors in each day with the total patterns of 16 patterns; watching television was the behavior performed at most (Lenz et al., 2014). It could reflect that the sedentary behaviors in the study participants may not be caused by not only social networks, but also other activities since the participants spent time on social networks quite the same as time spent to perform other any activities. This might be the reason why there was no relationship was found between the social network and the sedentary behaviors in this study. However, the limitation of this study finding should be acknowledged since the participants were the older adults living in the big city and its surrounding areas; a similar study conducted on the older adults living in the countryside in order to generalize the finding is recommended. Besides, the result of the program handling with depressive symptoms should be performed to encourage the physical activities in older adults.

\section{CONCLUSION}

Due to technological advances bring about physical and mental health impact, this study provides healthcare providers to understand social networking behaviors, depressive symptom, and sedentary behavior in older adults along with their association. These could help healthcare providers to plan further any appropriate interventions to promote healthy behavior in older adults.

\section{ACKNOWLEDGEMENT}

This study was supported by the Faculty of Medicine Ramathibodi Hospital, Mahidol University, Thailand to be presented at the 11th International Association of Gerontology and Geriatrics Asia/Oceania Regional Congress, 23-27 October, 2019, Taipei, Taiwan. Also, we deeply appreciated the participants who willing to share their information and time to participate in this study.

\section{REFERENCES}

Akeplakorn W, 2015. Report of the 5th Thai Health Survey by Physical Exam 2014. Health Systems Research Institute, Nonthaburi. pp. 7888.

Berkman LF, Glass T, Brissette I, Seeman TE, 2000. From social integration to health: Durkheim in the new millennium. Soc Sci Med, 51: 843-857.

Bishwajit G, O'Leary DP, Ghosh S, Yaya S, Shangfeng T, Feng Z, 2017. Physical inactivity and self-reported depression among middleand older-aged population in South Asia: World health survey. BMC Geriatr, 17: 1-8.

Borson S, Scanlan JM, Chen P, Ganguli M, 2003. The Mini-Cog as a screen for dementia: validation in a population-based sample. J Am Geriatr Soc, 51: 1451-1454.

Buntadthong $\mathrm{K}, 2014$. Social network usage behavior and Bangkok older person's satisfaction. Unpublished dissertation in partial fulfillment of the requirements for the master of Business Administration, Bangkok University, Bangkok, Thailand.

Carmpbell CM, 2012. Methods of physical activity assessment in older adults. Iowa State University, lowa. https://lib.dr.iastate.edu/etd/ 12809 (Accessed on August 15, 2018).

Chartprasert D, 2013. Users' behaviors and uses of Facebook. J Hum Soc Buu, 21: 35-57.

Chopik WJ, 2016. The benefits of social technology use among older adults are mediated by reduced loneliness. Cyberpsych Beh Soc N, 19: 551-556.

Cotton SR, Ford G, Ford S, Hale TM, 2014. Internet use and depression among retired older adults in the United States: A longitudinal analysis. Gerontologist, 15: 666-675.

De Rezende LF, Rey-López JP, Matsudo VKR, do Carmo-Luiz O, 2014. Sedentary behavior and health outcomes among older adults: a systematic review. BMC Public Health, 14: 333.

Elliot AJ, Mooney CJ, Douthit KZ, Lynch MF, 2014. Predictors of older adults' technology use and its relationship to depressive symptoms and well-being. J Gerontol B Psychol Sci Soc Sci, 69: 667-77.

Faul $F$, Erdfelder E, Buchner A, Lang AG, 2009. Statistical power analyses using $G^{*}$ Power 3.1: tests for correlation and regression analyses. Behav Res Methods, 41: 1149-1160.

Gell NM, Rosenberg DE, Demiris G, LaCroix AZ, Patel KV, 2015. Patterns of technology use among older adults with and without disabilities. Gerontologist, 55: 412-421.

Heo J, Chun S, Lee S, Lee KH, Kim J, 2015. Internet use and wellbeing in older adults. Cyberpsych Beh Soc N, 18: 268-272.

Koocharoensin S, 2016. The study of using social media behavior of the elderly in Muang district Chonburi province. Unpublished dissertation in partial fulfillment of the requirements for the master of Education Program. Burapha University, Chonburi, Thailand.

Lenz E, Swartz A, Strath S, 2014. Do sedentary behaviors modify the health status of older adults? Int J Kinesiology Sport Sci, 2: 13-22.

Loipah S, 2011. The elderly and information technology. J InfSci, 29: 53-64.

M'hiri K, Costanza A, Khazaal Y, Khan R, Zullino D, Achab S, 2015. Problematic internet use in older adults, a critical review of the literature. J Addict Res Ther, 6: 253.

National Statistical Office Ministry of Information and Communication Technology, 2017. The 2017 Household Survey on the Use of Information and Communication Technology. Bangkok, Thailand. http://service.nso.go.th (Accessed on April 18, 2018).

Oner K, Arslantas H, 2016. Depression, internet addiction and Ioneliness relations in adolescents of high school students. JMB, 2: 45-51.

Pearson N, Braithwaite R, Biddle SJ, van Sluijs EM, Atkin AJ, 2014. Associations between sedentary behavior and physical activity in children and adolescents: a meta-Pedometer accuracy in slowwalking older adults. Int J Therapy Rehabil, 19: 387-393.

Penninx BW Kritchevsky SB, Yaffe K, Newman AB, Simonsick EM, Rubin S, Ferrucci L, Harris T, Pahor M, 2003. Inflammatory markers and depressed mood in older persons: results from the health, aging and body composition study. Biol Psychiat, 54: 566-572.

Perrin A, 2015. Social media usage: 2005-2015. Pew Research Center, United States of America. http://www.pewinternet.org/2015/10/08/ 2015/Social-Networking-Usage-2005-2015/ (Accessed on May 5, 2018).

Pomnu S, Roonpho P, 2017. Behavior using social networks to promote the quality of life for elderly. Faculty of Management Sciences, Silpakorn University, Bangkok, Thailand http://repository.rmutr.ac.th (Accessed on April 20, 2018).

Poolsawat V, 2007. Physical activity of the older adults in Bangkok. Unpublished dissertation in partial fulfillment of the requirements for the master of Nursing Science, Mahidol University, Nakhon Pathom, Thailand.

Reynolds K, Pietrzak RH, El-Gabalawy R, Mackenzie CS, Sareen J, 2015. Prevalence of psychiatric disorders in US older adults: findings from a nationally representative survey. World Psychiatry, 14: 74-81.

Rodda J, Walker Z, Carter J, 2011. Depression in older adults. Brit Med J, 343: 683-687. 
Rosenberg DE, Bellettiere J, Gardiner PA, Villarreal VN, Crist K, Kerr J, 2015. Independent associations between sedentary behaviors and mental, cognitive, physical, and functional health among older adults in retirement communities. J Gerontol: series A, 71: 78-83.

Schuch F Vancampfort D, Firth J, Rosenbaum S, Ward P, Reichert T, Bagatini NC, Bgeginski R, Stubbs B, 2017. Physical activity and sedentary behavior in people with major depressive disorder: a systematic review and meta-analysis. J Affect Disorders, 210: 139150.

Senkaew K, 2015. Social Network Usage Behaviours of X Generation in Bangkok. Unpublished dissertation in partial fulfillment of the requirements for the master of Business Administration, Bangkok University, Bangkok, Thailand.

Shankar A, McMunn A, Demakakos P, Hamer M, Steptoe A, 2017. Social isolation and loneliness: Prospective associations with functional status in older adults. Health Psychol, 36: 179.

The Institute for Population and Social Research, 2016. Survey of physical activity of the population 2012-2016, Mahidol University, NakhonPathom, Thailand.

Thiengtham S, 2015. Physical activity, nutritional status and depression related to sarcopenia in community dwelling older adults. Unpublished dissertation in partial fulfillment of the requirements for the master of Nursing Science. Mahidol University, NakhonPathom, Thailand.

Trongsakul S, 2013. The prevalence of undiagnosed cognitive impairment and prevalence of undiagnosed depressive mood in over 60's with type 2 diabetes in a Thai community: a cross-sectional study. Unpublished dissertation in partial fulfillment of the requirements for the degree of Medicine and Health Sciences, University of East Anglia, Norwich, United Kingdom.

Vallance JK, Eurich DT, Lynch BM, Gardiner PA, Taylor LM, Jefferis BJ, Johnson ST, 2016. Correlates of general and domain-specific sitting time among older adults. Am J Health Behav, 40: 362-370.

Wongpakaran N, Wongpakaran T, Reekum RV, 2013. The use of GDS-15 in detecting MDD: a comparison between residents in a Thai long-term care home and geriatric outpatients. J Clin Med Res, 5: $101-111$.

World Health Organization (WHO), 2017. Depression and other common mental disorders: Global Health Estimates. Geneva, Switzerland. https://apps.who.int/iris/bitstream/handle/10665/254610 WHO-MSD-MER-2017.2-eng.pdf (Accessed on May 10, 2018).
Citation: Baurangtheinthong S, Aree-Ue S, Leelacharas S, 2019. Relationships among social networking behaviors, depressive symptom and sedentary behaviors in older adults. Int Res J Med Med Sci, 8(1): 1-7. 